\title{
Heat transfer and phase change during picosecond laser ablation of nickel
}

\author{
David A. Willis ${ }^{1}$, Xianfan $\mathrm{Xu}{ }^{*}$ \\ School of Mechanical Engineering, Purdue University, 1288 Mechanical Engineering Building, West Lafayette, IN 47907, USA
}

Received 17 April 2001; received in revised form 9 March 2002

\begin{abstract}
This work investigates heat transfer and phase change during picosecond laser ablation of nickel. In this study, ablation of nickel is studied using a mode-locked 25 ps (FWHM) Nd:YAG laser. The threshold fluence for mass removal (ablation) is experimentally determined. Numerical calculations of the transient temperature distribution and kinetics of the solid-liquid and liquid-vapor phase change interfaces are performed. The results show that evaporation is negligible at the free surface, resulting in superheating of liquid to near $0.9 T_{\text {cr }}$, at which temperature homogeneous nucleation will result in an explosive phase transformation, removing part of the molten layer. (C) 2002 Elsevier Science Ltd. All rights reserved.
\end{abstract}

Keywords: Picosecond laser; Homogeneous nucleation; Explosive phase transformation; Heat transfer

\section{Introduction}

Short-pulse laser micromachining has attracted much attention due to the ability to fabricate microstructures with high precision. Applications of short-pulse laser micromachining include fabrication of medical devices, microsensors [1], and microelectronics [2]. Picosecond $\left(10^{-12} \mathrm{~s}\right)$ and femtosecond $\left(10^{-15} \mathrm{~s}\right)$ lasers are of great interest for micromachining since the reduced pulse widths result in a reduction of thermal diffusion length. In order to control and optimize the laser-induced material removal during micromachining, it is necessary to understand the energy transport and phase change processes. The thermal processes resulting in mass removal during picosecond and femtosecond laser-material interactions are not completely understood $[3,4]$.

During short-pulse laser heating of metals, the laserirradiated surface will easily exceed the melting temperature, and for high fluences the normal boiling

\footnotetext{
${ }^{*}$ Corresponding author. Tel.: +1-765-494-5639; fax: +1-765494-0539.

E-mail address: xxu@ecn.purdue.edu (X.Xu).

${ }^{1}$ Current address: Department of Mechanical Engineering, Southern Methodist University, Dallas, TX 75275.
}

temperature will be reached. To illustrate what occurs in the laser-melted layer, the $P-T$ diagram including the binode and the spinode for liquid-vapor phase change of a typical metal is shown in Fig. 1. Data are available for cesium but it is believed that other metals follow the same trend. The binode is the equilibrium liquid-vapor line observed under slow heating. At low temperatures and slow heating, this line may be calculated from the Clausius-Clapeyron equation. Under such slow heating conditions, only small superheating is allowed past the binode since excess energy will result in the growth of heterogeneous nuclei rather than further heating of the liquid. If the heating rate is rapid enough $\left(>10^{9} \mathrm{~K} / \mathrm{s}\right)$ the molten surface may be superheated significantly past the normal boiling point, since heterogeneous nuclei will not have sufficient time for growth [5]. Superheating may be deep enough that the liquid approaches the kinetic limit of superheat, or the spinode, which is also shown in Fig. 1. The stability of a liquid can be described by "stability coefficients" given by [5]:

$$
\begin{aligned}
& \left(\frac{\partial P}{\partial V}\right)_{\mathrm{T}}=\frac{K T}{\overline{V^{2}}-\bar{V}^{2}}, \\
& \left(\frac{\partial T}{\partial S}\right)_{\mathrm{P}}=\frac{K T^{3}}{\overline{H^{2}}-\bar{H}^{2}} .
\end{aligned}
$$




\begin{tabular}{|c|c|c|c|}
\hline \multicolumn{4}{|c|}{ Nomenclature } \\
\hline$B$ & melt interface kinetic constant (m/s K) & $x$ & coordinate perpendicular to target surface \\
\hline$c_{\mathrm{p}}$ & specific heat at constant pressure $(\mathrm{J} / \mathrm{kg} \mathrm{K})$ & & (m) \\
\hline$f_{1}$ & liquid fraction of finite difference cell & \multicolumn{2}{|c|}{ Greek symbols } \\
\hline$f_{\mathrm{v}}$ & vapor fraction of finite difference cell & $\alpha$ & thermal diffusivity $\left(\mathrm{m}^{2} / \mathrm{s}\right)$ \\
\hline$K$ & Boltzmann's constant $(\mathrm{J} / \mathrm{K})$ & & sticking coefficient \\
\hline$k$ & thermal conductivity (W/m K) & $\Delta H_{\mathrm{sl}}$ & enthalpy of fusion $(\mathrm{J} / \mathrm{kg})$ \\
\hline $\begin{array}{l}H \\
h\end{array}$ & $\begin{array}{l}\text { enthalpy }(\mathrm{J} / \mathrm{kg} \mathrm{K}) \\
\text { volumetric enthalpy }\left(\mathrm{J} / \mathrm{m}^{3} \mathrm{~K}\right)\end{array}$ & $\Delta H_{\mathrm{lv}}$ & enthalpy of vaporization $(\mathrm{J} / \mathrm{kg})$ \\
\hline I & laser irradiance $\left(\mathrm{W} / \mathrm{m}^{2}\right)$ & $\Delta t$ & time step (s) \\
\hline j & molar mass flux $\left(\mathrm{kmol} / \mathrm{m}^{2} \mathrm{~s}\right)$ & $\mu$ & absorption coefficient $\left(\mathrm{m}^{-1}\right)$ \\
\hline l & length $(\mathrm{m})$ & $\rho$ & density $\left(\mathrm{kg} / \mathrm{m}^{3}\right)$ \\
\hline$M$ & molar weight $(\mathrm{kg} / \mathrm{kmol})$ & $\tau$ & laser pulse width $(\mathrm{s})$ \\
\hline$P$ & pressure $\left(\mathrm{N} / \mathrm{m}^{2}\right)$ & \multicolumn{2}{|c|}{ Subscripts } \\
\hline$P_{\text {sat }}$ & saturation pressure $\left(\mathrm{N} / \mathrm{m}^{2}\right)$ & $\mathrm{amb}$ & ambient conditions \\
\hline$\dot{q}$ & heat generation $\left(\mathrm{W} / \mathrm{m}^{3}\right)$ & $\mathrm{i}$ & finite difference node index \\
\hline$R$ & universal gas constant $(\mathrm{J} / \mathrm{kmol} \mathrm{K})$ & 1 & liquid \\
\hline$r$ & reflectivity & $\mathrm{lv}$ & liquid-vapor interface \\
\hline$S$ & entropy $(\mathrm{J} / \mathrm{kg})$ & 0 & absolute zero temperature conditions \\
\hline$T$ & temperature $(\mathrm{K})$ & s & solid \\
\hline$T_{\mathrm{b}}$ & equilibrium boiling temperature $(\mathrm{K})$ & sl & solid-liquid interface \\
\hline$T_{\mathrm{cr}}$ & thermodynamic critical temperature $(\mathrm{K})$ & th & thermal \\
\hline$T_{\mathrm{m}}$ & equilibrium melting temperature $(\mathrm{K})$ & \multirow{2}{*}{\multicolumn{2}{|c|}{ Superscripts }} \\
\hline$t$ & time (s) & & \\
\hline$u$ & interface velocity $(\mathrm{m} / \mathrm{s})$ & new & current time step in numerical calculation \\
\hline$V$ & volume $\left(\mathrm{m}^{3}\right)$ & old & previous time step in numerical calculation \\
\hline
\end{tabular}



Fig. 1. Pressure-temperature diagram for cesium.

These stability coefficients are inversely proportional to volume and enthalpy fluctuations, where the bar over the volume and enthalpy values denotes a time average of a fluctuation. As a superheated liquid approaches the critical temperature, these fluctuations become very large, resulting in the value of these coefficients approaching zero. The location where the coefficients are zero defines the spinode, thus the spinodal curve can be found from Eqs. (1) and (2) if the equation of state is known. The spinodal curve shown in Fig. 1 is obtained from experimental data in the literature [5], which shows that the limit of superheat for the short-pulse laser heating process is approximately $0.9 T_{\mathrm{cr}}$. The exact temperature-pressure relationship is not known, but the pressure is expected to be only slightly higher than atmospheric pressure since equilibrium conditions are not achieved [6]. As the liquid temperature approaches the spinode, fluctuations in density become large enough that regions of low density will form vapor nuclei. The rate of formation of vapor nuclei increases exponentially near the spinode, resulting in homogeneous boiling of the liquid $[3,7,8]$, also called explosive phase transformation or phase explosion. These large fluctuations in density also result in a dramatic increase in electrical resistivity and specific heat, as seen in Fig. 2. The increase in electrical resistivity is commonly referred to as the "dielectric transition", and results in the surface becoming optically transparent [7]. Experimental evidence of laser-induced phase explosion was first obtained during the study of laser ablation of nickel with a $26 \mathrm{~ns}$ pulsed excimer laser [8]. Superheating and the departure of the surface temperature-pressure relation 


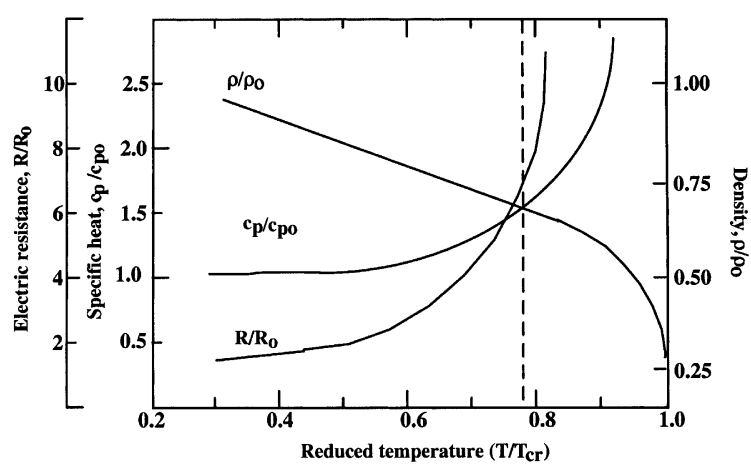

Fig. 2. Typical variation of properties for a liquid metal.

from the Clausius-Clapeyron relation were also demonstrated for nanosecond pulsed excimer laser ablation of nickel $[6,9]$.

This study is an experimental and numerical investigation of picosecond laser ablation of metal. Experiments are performed using a mode-locked 25-ps Nd:YAG laser and nickel targets. The fluence at the ablation threshold is determined, and the ablation depth as a function of fluence is determined for fluences above the ablation threshold. Numerical calculations are performed to determine the temperature field, melt depth, and amount of mass removed by surface vaporization. These calculations provide insight to the thermal mechanisms responsible for mass removal.

\section{Experimental study}

An experimental parametric study is performed to determine the ablation threshold and the ablation depth as a function of laser fluence. Experiments are performed using a mode-locked Nd:YAG laser with a pulse width of 25 ps (FWHM) operating at a pulse repetition rate of $10 \mathrm{~Hz}$. The laser wavelength is $1064 \mathrm{~nm}$. The incident beam energy is manipulated by a series of optical waveplates and polarizers. The laser spot size is adjusted by changing the distance between the focusing lens and the target. A polished nickel sample $(5 \mathrm{~nm}$ RMS roughness) is ablated with multiple pulses of the laser and the final depth is measured with a stylus profilometer. The ablation depth per pulse is obtained by dividing the final ablated depth by the number of incident pulses. The exact number of pulses for each hole is counted by reflecting a portion of the laser to a fast photodiode, which is connected to an electronic counter that records the number of times the photodiode is triggered.

The resulting ablation depth as a function of laser fluence is shown in Fig. 3 for the ablation of nickel in air at atmospheric conditions. The fluence is estimated from the measured pulse energy and the diameter of the ab-

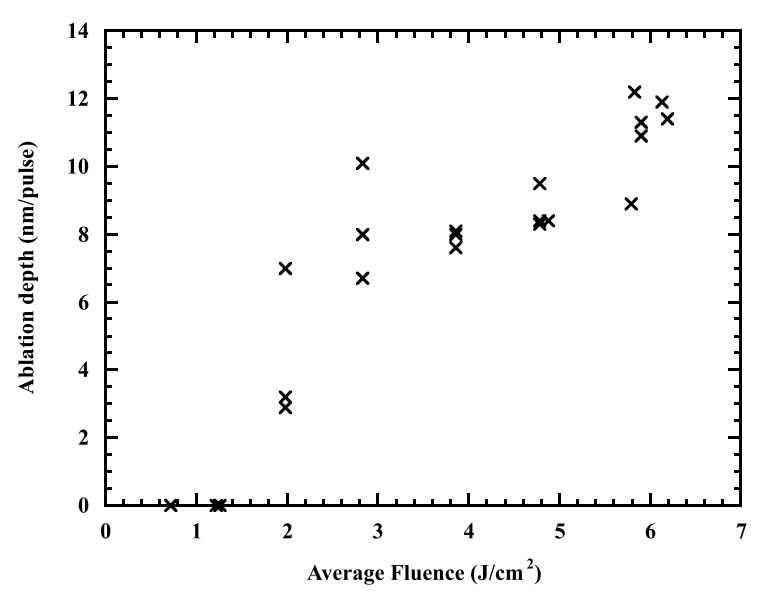

Fig. 3. Ablation depth vs. incident laser fluence for ablation of nickel in air.

lated area. The diameter of the ablated area was measured with an optical microscope to be approximately $150 \mu \mathrm{m}$. The resulting holes are tapered, and the ablation depth in Fig. 3 is the maximum depth measured in the center of the hole. At the threshold fluence the removal rate is between 3 and $7 \mathrm{~nm} /$ pulse. Above the ablation threshold the ablation rate does not increase rapidly, and there is considerable scatter in the data. A visible plume of material is observed at fluences above the ablation threshold. This is due to the extremely high temperature reached in the ablated materials and in the adjacent air, and possibility electron emission from the surface [10,11]. Although an accurate estimate of the laser fluence was not available, the data are useful for demonstrating the threshold nature of the ablation process. This will be discussed in more detail later in this work.

\section{Numerical calculation}

Numerical modeling is performed to calculate the transient heat transfer and phase change during the laser ablation process. Reflection of the laser beam and volumetric absorption of the laser energy are considered. Melting and vaporization kinetics are also included in the model to predict the depth of melting and amount of mass lost to evaporation at the free surface.

\subsection{Governing equations and phase change interface kinetics}

The transient temperature field is obtained by solving the one-dimensional heat diffusion equation, based on Fourier's law of conduction: 
$\rho c_{\mathrm{p}} \frac{\partial T}{\partial t}=\frac{\partial}{\partial x}\left(k \frac{\partial T}{\partial x}\right)+\dot{q}$,

where $k$ is the thermal conductivity, $\rho$ is the density, $c_{\mathrm{p}}$ is the specific heat, $T$ is the temperature, and $\dot{q}$ is the volumetric heat generation. Heat generation is governed by the absorption of laser energy within the absorption depth:

$\dot{q}=-\frac{\mathrm{d} I(x, t)}{\mathrm{d} x}=\mu(1-r) I(t) \mathrm{e}^{-\mu x}$,

where $\mu$ is the absorption coefficient, $x$ is the distance from the free surface, $I(t)$ is the incident laser irradiance, and $r$ is the nickel reflectivity.

The energy balance at the solid-liquid interface is

$\rho_{1} \Delta H_{\mathrm{sl}} u_{\mathrm{sl}}=k_{\mathrm{s}} \frac{\partial T_{\mathrm{s}}}{\partial x}-k_{1} \frac{\partial T_{1}}{\partial x}$,

where $\rho_{1}$ is the liquid density, $k_{\mathrm{s}}$ is the solid phase thermal conductivity, and $k_{1}$ is the liquid phase thermal conductivity. During fast melting and solidification, the rate of melting may be limited by interface kinetics rather than heat flow at the interface. For metals, interface kinetics is described by a collision-limited interface growth model [12]. For collision limited growth the solid-liquid interface velocity, $u_{\mathrm{sl}}$, is proportional to the interface superheating [13]

$u_{\mathrm{sl}}=B\left(T_{\mathrm{sl}}-T_{\mathrm{m}}\right)$,

where $T_{\mathrm{sl}}$ is the solid-liquid interface temperature, $T_{\mathrm{m}}$ is the equilibrium melting temperature, and $B$ is a kinetic melting coefficient, which is constant for low superheating.

The boundary condition at the free surface is governed by the evaporative flux of atoms, and the mathematical expression for this energy balance at the free surface is given as

$\left.k_{1} \frac{\partial T}{\partial x}\right|_{x=0}=M j \Delta H_{\mathrm{lv}}$,

where $M$ is the molar weight of nickel, $\Delta H_{\mathrm{lv}}$ is the enthalpy of vaporization at the liquid-vapor interface temperature, $T_{\mathrm{lv}}$, which is a function of temperature given by [14]

$\Delta H_{\mathrm{lv}}=\Delta H_{\mathrm{lv}, 0}\left[1-\frac{T_{\mathrm{lv}}^{2}}{T_{\mathrm{cr}}^{2}}\right]^{1 / 2}$,

where $T_{\mathrm{cr}}$ is the thermodynamic critical temperature and $\Delta H_{\mathrm{lv}, 0}$ is the enthalpy of vaporization at absolute zero. In Eq. (7) $j$ is the molar flux of atoms at the free surface, and is described by the Hertz-Knudsen equation [15] $j=\frac{0.82 \beta\left(P_{\mathrm{sat}}-P_{\mathrm{amb}}\right)}{\left(2 \pi M R T_{\mathrm{lv}}\right)^{1 / 2}}$,

where $\beta$ is the sticking coefficient, which is approximately unity for metals [15]. $P_{\mathrm{amb}}$ is the ambient pressure, $P_{\text {sat }}$ is the saturation pressure at the liquid-vapor interface temperature and $R$ is the universal gas constant. The coefficient, 0.82, in Eq. (9) is the result of a back-flow of approximately $18 \%$ of the evaporated atoms to the surface, which is obtained from solving the conservation equations [16]. The saturation pressure is given by a modified Clausius-Clapeyron equation, which incorporates the temperature dependence of the enthalpy of vaporization [17]

$$
\begin{aligned}
P_{\text {sat }}(T)=P_{\text {amb }} \exp \left\{\frac { - \Delta H _ { \mathrm { lv } , 0 } M } { R } \left[\frac{1}{T_{\mathrm{lv}}}\left(1-\left(\frac{T_{\mathrm{lv}}}{T_{\mathrm{cr}}}\right)^{2}\right)^{1 / 2}\right.\right. \\
\left.\quad-\frac{1}{T_{\mathrm{b}}}\left(1-\left(\frac{T_{\mathrm{b}}}{T_{\mathrm{cr}}}\right)^{2}\right)^{1 / 2}\right]-\frac{\Delta H_{\mathrm{lv}, 0} M}{R T_{\mathrm{cr}}}\left[\sin ^{-1}\left(\frac{T_{\mathrm{lv}}}{T_{\mathrm{cr}}}\right)\right. \\
\left.\left.-\sin ^{-1}\left(\frac{T_{\mathrm{b}}}{T_{\mathrm{cr}}}\right)\right]\right\} .
\end{aligned}
$$

The far-field boundary condition is given by a zero temperature gradient since the size of the numerical domain $(1 \mu \mathrm{m})$ is much larger than the estimated thermal diffusion depth of approximately $24 \mathrm{~nm}$. The thermal diffusion length is estimated from

$l_{\mathrm{th}}=(\alpha \tau)^{1 / 2}$,

where $\alpha$ is the thermal diffusivity and $\tau$ is the full pulse width (50 ps) of the laser. The diameter of the focused laser beam was $150 \mu \mathrm{m}$, which is much larger than the estimate thermal diffusion length, justifying the onedimensional approximation.

\subsection{Numerical technique}

Eqs. (3)-(10) are solved numerically using a finite difference scheme with an implicit time integration technique. The diffusion equation is solved using the enthalpy technique, which eliminates the need for explicit tracking of the solid-liquid interface location. The enthalpy form of the diffusion equation is given as

$\frac{\partial h}{\partial t}=\frac{\partial}{\partial x}\left(k \frac{\partial T}{\partial x}\right)+\dot{q}$,

where $h$ is the enthalpy per unit volume. The enthalpytemperature relation for a finite-difference cell is given as

$h=\int_{T_{\mathrm{amb}}}^{T} \rho c_{\mathrm{p}} \mathrm{d} T+f_{1} \rho_{1} \Delta H_{\mathrm{sl}}+f_{\mathrm{v}} \rho_{1} \Delta H_{\mathrm{lv}}$,

where $f_{1}$ and $f_{\mathrm{v}}$ are the liquid and vapor fractions, respectively, for a node. A fixed grid scheme is used for the 
calculations. Vaporization is handled by giving the vapor cells the same temperature as the liquid-vapor interface to prevent conduction into the vapor. The iteration procedure is as follows:

(1) The initial temperature field is set to the ambient temperature. Eqs. (12) and (13) are solved for the enthalpy in each node.

(2) When the equilibrium melting temperature is reached, the solid-liquid interface temperature, $T_{\mathrm{sl}}$, is given an assumed temperature higher than the equilibrium value, and the liquid fraction is computed for each node. Steps (1) and (2) are repeated until the enthalpy converges according to

$\left|\frac{h_{\mathrm{i}}^{\text {new }}-h_{\mathrm{i}}^{\text {old }}}{h_{\mathrm{i}}^{\text {old }}}\right|<10^{-14}$,

where $h_{\mathrm{i}}^{\text {new }}$ and $h_{\mathrm{i}}^{\text {old }}$ are the enthalpy of the node at the current time step $(t)$ and the previous time step $(t-1)$.

(3) Using the converged value of the liquid fraction, the location of the interface is calculated and the interface velocity is computed from

$u_{\mathrm{sl}, \mathrm{int}}=\frac{x_{\mathrm{sl}}^{\mathrm{new}}-x_{\mathrm{sl}}^{\mathrm{old}}}{\Delta t}$,

where $x_{\mathrm{sl}}^{\text {new }}$ and $x_{\mathrm{sl}}^{\text {old }}$ are the solid-liquid interface locations at the current time step $(t)$ and the previous time step $(t-1)$. This interfacial velocity is then used to compute a new interface temperature using Eq. (6). If the new interface temperature differs from the value assumed in Step (2), iterations are carried out until the interface temperatures calculated from two successive iterations satisfy the convergence criterion,

$$
\left|T_{s l}^{\text {new }}-T_{s l}^{\text {old }}\right|<5 \times 10^{-5} .
$$

(4) For the computation of the liquid-vapor interface temperature and kinetics, a similar procedure to Step (3) is performed.

Temperature dependent thermophysical properties are used and are given in Table 1. A domain of $1 \mu \mathrm{m}$ was modeled using 150 finite difference cells. The cells are graded such that smaller cells are located at the free surface where the laser energy is absorbed. The temporal distribution of the laser pulse intensity is approximated as a triangular. Optical properties are also listed in Table 1 and are calculated from the refractive index data for nickel [18].

\subsection{Numerical results}

The transient surface temperature as a function of incident laser fluence is shown in Fig. 4. The threshold fluence for reaching the melting temperature is $102 \mathrm{~mJ} /$ $\mathrm{cm}^{2}$. Above $300 \mathrm{~mJ} / \mathrm{cm}^{2}$, the molten nickel is heated above the equilibrium boiling point ( $3188 \mathrm{~K})$. As explained previously, volumetric boiling due to heterogeneous nucleation is not likely to occur however, since the nuclei do not have enough time to grow to critical size. At a fluence of $508 \mathrm{~mJ} / \mathrm{cm}^{2}$, the surface has reached 6100 $\mathrm{K}$, which is approximately $0.78 T_{\mathrm{cr}}$. Numerical modeling was not performed past $508 \mathrm{~mJ} / \mathrm{cm}^{2}$, since at $0.78 T_{\text {cr }}$ or higher temperatures large fluctuations in thermal and optical properties will occur due to large homophase fluctuations, as shown in Fig. 2.

The transient melt depth as a function of laser fluence is shown in Fig. 5. The melt depth continues to increase

Table 1

Thermophysical and optical properties of nickel [7,15,17,18,20-22]

\begin{tabular}{|c|c|c|}
\hline Solid thermal conductivity $(\mathrm{W} / \mathrm{m} \mathrm{K})$ & $\begin{array}{l}298 \mathrm{~K}<T<600 \mathrm{~K} \\
600 \mathrm{~K}<T<1726 \mathrm{~K}\end{array}$ & $\begin{array}{l}k_{\mathrm{s}}=114.43-0.082 T \\
k_{\mathrm{s}}=50.47+0.012 T \\
k_{1}=89\end{array}$ \\
\hline Solid specific heat $(\mathrm{J} / \mathrm{kg} \mathrm{K})$ & $298 \mathrm{~K}<T<1400 \mathrm{~K}$ & $\begin{array}{l}c_{\mathrm{p}, \mathrm{s}}=-295.95+4.95 T-0.0096 T^{2}+ \\
9.46 \times 10^{-6} T^{3}-4.36 \times 10^{-9} T^{4}+7.60 \times 10^{-13} T\end{array}$ \\
\hline & $T>1400 \mathrm{~K}$ & $c_{\mathrm{p}, \mathrm{s}}=616.56$ \\
\hline Liquid specific heat $(\mathrm{J} / \mathrm{kg} \mathrm{K})$ & & $c_{\mathrm{p}, 1}=734.16$ \\
\hline Enthalpy of fusion $(\mathrm{J} / \mathrm{kg})$ & & $\Delta H_{\mathrm{sl}}=298.3$ \\
\hline Enthalpy of vaporization $(\mathrm{J} / \mathrm{kg})$ & & $\Delta H_{\mathrm{lv}, 0}=6453.2$ \\
\hline Equilibrium melting temperature $(\mathrm{K})$ & & $T_{\mathrm{m}}=1726$ \\
\hline Equilibrium boiling temperature $(\mathrm{K})$ & & $T_{\mathrm{b}}=3188$ \\
\hline Critical temperature $(\mathrm{K})$ & & $T_{\mathrm{cr}}=7810$ \\
\hline Molar weight (kg/kmol) & & $M=58.7$ \\
\hline Density $\left(\mathrm{kg} / \mathrm{m}^{3}\right)$ & & $\rho=8900$ \\
\hline Sticking coefficient & & $\beta=1$ \\
\hline Melt interface kinetic constant $(\mathrm{m} / \mathrm{s} \mathrm{K})$ & & $B=1.18$ \\
\hline Nickel reflectivity & & $r=0.7255$ \\
\hline Absorption coefficient $\left(\mathrm{m}^{-1}\right)$ & & $\mu=6.138 \times 10^{7}$ \\
\hline
\end{tabular}




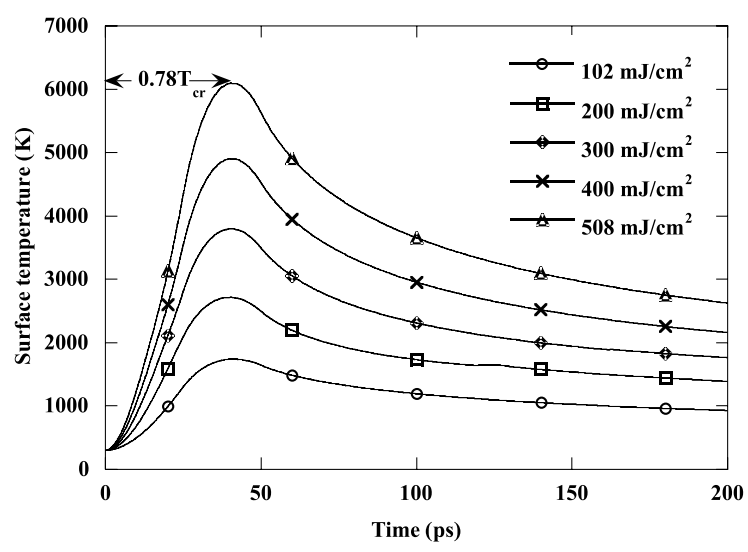

Fig. 4. Transient surface temperature vs. incident laser fluence.

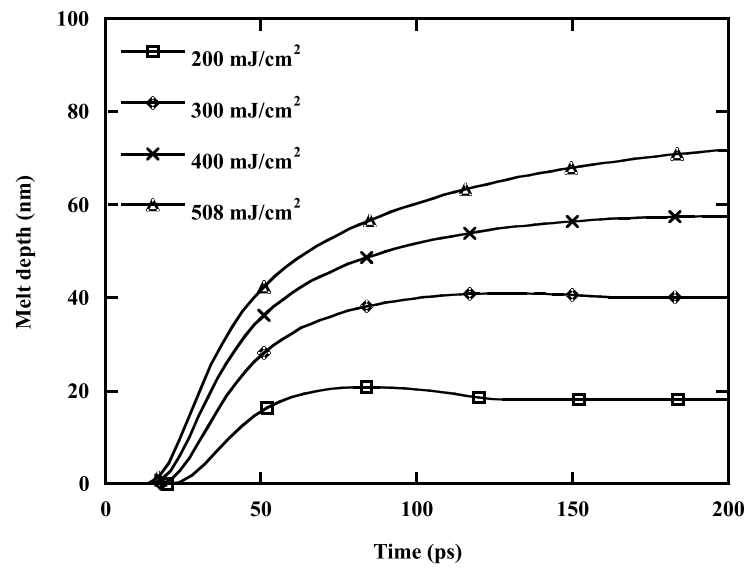

Fig. 5. Transient melt depth vs. incident laser fluence.

after the laser pulse has ended at $50 \mathrm{ps}$ due to heat diffusion away from the near surface region. At the highest fluence used, the maximum melt depth has reached 70 $\mathrm{nm}$ at $200 \mathrm{ps}$, while the depth of material removed by evaporation is less than $0.1 \mathrm{~nm}$, as shown in Fig. 6. The temperature as a function of distance from the free surface is shown in Fig. 7 for a laser fluence of $508 \mathrm{~mJ} /$ $\mathrm{cm}^{2}$. This plot demonstrates that a domain size of $1 \mu \mathrm{m}$ is sufficient to properly model the heat transfer and phase change, since the temperature is reduced to ambient temperature at a distance of less than $250 \mathrm{~nm}$ from the free surface.

\section{Discussion}

Experimental results demonstrate the threshold nature of the ablation process. Above the threshold fluence, significant ablation on the order of a few nanometers per pulse or more is obtained. On the other

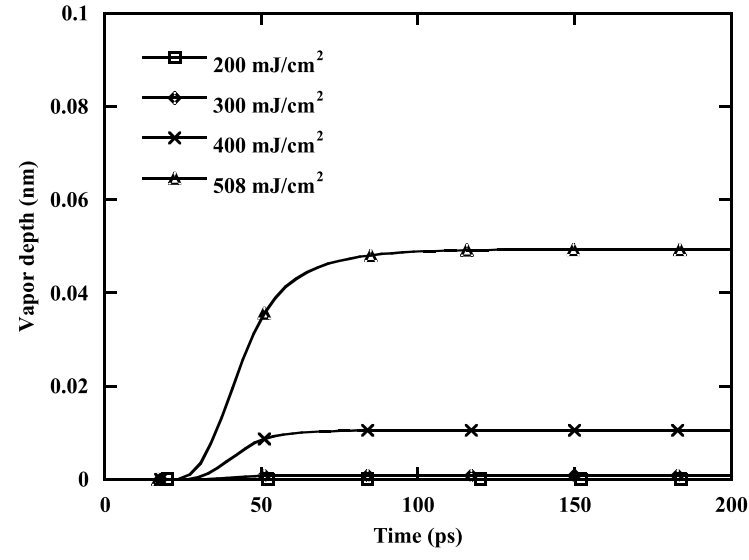

Fig. 6. Transient vapor depth vs. incident laser fluence.

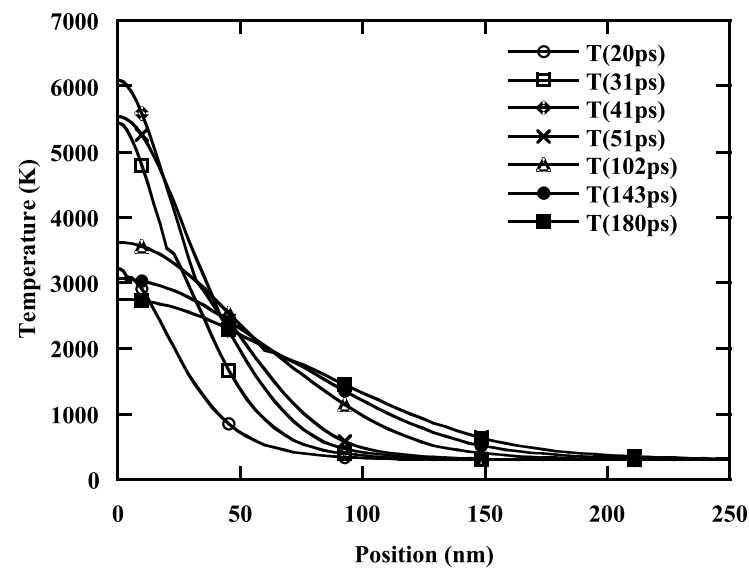

Fig. 7. Transient temperature as a function of distance from free surface, $F=508 \mathrm{~mJ} / \mathrm{cm}^{2}$.

hand, numerical modeling shows no significant surface evaporation. The evaporation depth is $<0.1 \mathrm{~nm}$ even when the surface temperature reaches $0.78 T_{\mathrm{cr}}$. This demonstrates that surface evaporation is not the mechanism causing significant mass removal. The ablation rate observed experimentally can only be explained as a result of phase explosion.

For a laser fluence of approximately five times higher than the melting threshold, the surface temperature of the liquid nickel is $0.78 T_{\text {cr. }}$. At this time ( $\left.40 \mathrm{ps}\right)$ this molten surface layer is approximately $40 \mathrm{~nm}$ thick. At higher laser fluences the surface temperature is expected to increase past $0.78 T_{\mathrm{cr}}$, and thermophysical property variations become extremely large and are not known accurately enough for numerical calculations. At temperatures even higher than $0.78 T_{\text {cr }}$ the large homophase fluctuations will become heterophase fluctuations, i.e. vapor nuclei will form, causing phase explosion at $0.9 T_{\text {cr }}$. When phase explosion occurs, both liquid and vapor are 
expected to be ejected, resulting in significant mass removal. The experimentally measured mass removal rate is less than the calculated melting depth, indicating that either not the entire layer of the liquid undergoes phase explosion, or the ablated material is partially redeposited to the laser irradiated area.

The quantitative comparison between the calculated ablation threshold of phase explosion (a bit higher than $\left.0.508 \mathrm{~J} / \mathrm{cm}^{2}\right)$ and measured ablation threshold $(\sim 2 \mathrm{~J} /$ $\left.\mathrm{cm}^{2}\right)$ is affected by uncertainties in both experiments and calculations, including uncertainty in the laser fluence, uncertainty in high-temperature thermophysical properties, and heat transfer model assumptions. The experimental laser fluence is an average fluence over the entire laser beam diameter, however the exact spatial irradiance distribution is unknown. It is likely that the irradiance will be higher at the center of the laser beam, therefore the threshold fluence may be higher than that reported. The high temperature thermophysical properties are not known for liquid nickel, therefore the values of the thermal conductivity and specific heat at the melting temperature are used. The numerical model assumes the classical Fourier's law description of the conduction process in the lattice. In reality, the electrons first absorb laser energy and then transfer this energy to the lattice on a time scale of a few picoseconds. Thus, there will be non-equilibrium effects due to the electronlattice coupling, resulting in higher electron temperatures and lower lattice temperatures than predicted by the model in this work [19]. More energy will be needed to raise the lattice temperature to the limit of superheat. The numerical model also assumed no absorption of laser energy above the nickel surface, however the work by Mao et al. $[10,11]$ indicates that plasma formation does occur during the laser pulse and interacts with the incident beam. Thus the numerical model will underestimate the threshold fluence for phase explosion. However, even if the factors mentioned above were all taken into account, the threshold behavior and the minimal mass removal due to surface evaporation would still result from the calculation.

\section{Conclusions}

The ablation threshold and the ablation depth as a function of laser fluence have been experimentally determined for ablation of nickel using a 25 ps Nd:YAG laser. Experiments demonstrated the threshold nature of the ablation process. Numerical modeling of the process shows that normal surface evaporation is not the mechanism of material removal, and that the molten surface is heated to near critical temperatures. Phase explosion is determined as the mechanism of material removal. The threshold obtained in experiments corresponds to the threshold for phase explosion.

\section{Acknowledgements}

Support to this work by the National Science Foundation and the Office of Naval Research is gratefully acknowledged.

\section{References}

[1] X. Xu, J.W. Ryu, D.A. Willis, Pulsed laser machining of thin films for microsensor development, in: Proceedings of the International Congress on Applications of Lasers and Electro-Optics, Vol. 88, Laser Microfabrication, Laser Institute of America, Orlando, FL, 1999, pp. 160-166.

[2] R.S. Patel, T.F. Redmond, C. Tessler, D. Tudryn, D. Pulaski, Laser via ablation technology for MCM-D fabrication at IBM Microelectronics, Int. J. Microcirc. Electron. Packaging 18 (3) (1995) 266-273.

[3] A. Miotello, R. Kelly, Critical assessment of thermal models for laser sputtering at high fluences, Appl. Phys. Lett. 67 (24) (1995) 3535-3537.

[4] A. Miotello, R. Kelly, Laser-induced phase explosion: new physical problems when a condensed phase approaches the thermodynamic critical temperature, Appl. Phys. A 69 (1999) S67-S73.

[5] M.M. Martynyuk, Vaporization and boiling of liquid metal in an exploding wire, Soviet Phys., Tech. Phys. 19 (6) (1974) 793-797.

[6] X. Xu, K. Song, Interface kinetics during pulsed laser ablation, Appl. Phys. A 69 (1999) S869-S873.

[7] M.M. Martynyuk, Critical constants of metals, Russ. J. Phys. Chem. 57 (4) (1983) 494-501.

[8] K.H. Song, X. Xu, Explosive phase transformation in excimer laser ablation, Appl. Surf. Sci. 127-129 (1998) 111116.

[9] X. Xu, K.H. Song, Phase change phenomena during high power laser-materials interaction, Mater. Sci. Eng. A 292 (2000) 162-168.

[10] S.S. Mao, X. Mao, R. Greif, R.E. Russo, Initiation of an early-stage plasma during picosecond laser ablation of solids, Appl. Phys. Lett. 77 (16) (2000) 2464-2466.

[11] S.S. Mao, X. Mao, R. Greif, R.E. Russo, Simulation of a picosecond laser ablation plasma, Appl. Phys. Lett. 76 (23) (2000) 3370-3372.

[12] M.J. Aziz, W.J. Boettinger, On the transition from shortrange diffusion-limited to collision-limited growth in alloy solidification, Acta Metall. Mater. 42 (2) (1994) 527-537.

[13] X. Xu, C.P. Grigoropoulos, R.E. Russo, Heat transfer in excimer laser melting of thin polysilicon layers, J. Heat Transfer 117 (1995) 708-715.

[14] B.S. Yilbas, Laser heating process and experimental validation, Int. J. Heat Mass Transfer 40 (1997) 11311143.

[15] M. von Allmen, A. Blatter, in: Laser-Beam Interactions with Materials, second ed., Springer-Verlag, Berlin, 1994, p. 119.

[16] S.I. Anisimov, Vaporization of metal absorbing laser radiation, Soviet Phys. JETP 27 (1) (1968) 182-183.

[17] X. Xu, G. Chen, K.H. Song, Experimental and numerical investigation of heat transfer and phase change phenomena 
during excimer laser interaction with nickel, Int. J. Heat Mass Transfer 42 (1999) 1371-1382.

[18] D.W. Lynch, W.R. Hunter, Comments on the optical constants of metals and an introduction to the data for several metals, in: E.D. Palik (Ed.), Handbook of the Optical Constants of Solids, Academic Press, Orlando, FL, 1985, p. 13.

[19] L.-S. Kuo, T. Qiu, Microscale energy transfer during picosecond laser melting of metal films, in: V. Prasad et al. (Eds.), Proceedings of the National Heat Transfer Confer- ence, HTD-vol. 323-1, ASME, New York, 1996, pp. 149156.

[20] F.P. Incropera, D.P. DeWitt, in: Fundamentals of Heat and Mass Transfer, third ed., John Wiley and Sons, New York, 1990, p. A5.

[21] I. Barin, in: Thermochemical Data of Pure Substances; Part II, VCH, New York, 1993, p. 1052.

[22] E.A. Brandes, G.B. Brook (Eds.), Smithells Metals Reference Book, seventh ed., Butterworth Heinemann, Oxford, 1992, pp. 14.1-14.13. 